\title{
Avaliação de pontas diamantadas sob influência da esterilização em autoclave
}

\section{Influence of the autoclave sterilization of diamond burs \\ Evaluación de puntas diamantadas bajo la influencia de la esterelización en autoclave}

Luanna Abílio Diniz Melquiades de MEDEIROS ${ }^{1}$

Elizandra Silva da PENHA2

Gymenna Maria Tenório GUÊNES ${ }^{3}$

Marcus Vinícius Lia FOOK ${ }^{4}$

Arthur Willian de Lima BRASIL ${ }^{5}$

Mylena de Araújo RÉ GIS ${ }^{\mathbf{6}}$

Thacyanna Wanderley JUCÁ

${ }^{1}$ Professora Doutora, Disciplinas de Clínica Multidisciplinar I e III, Estágio em Clínica Multidisciplinar Ie

Pré-Clínica Multidisciplinar V do curso de Odontologia da Universidade Federal de Campina Grande (UFCG), Unidade Acadêmica de Ciências Biológicas (UACB)58708-110, Patos-PB, Brasil

${ }_{2}^{2}$ Professora Mestre, Disciplinas de Clínica Infantil I e II, do curso de Odontologia da Universidade Federal de Campina Grande (UFCG), Unidade Acadêmica de Ciências Biológicas (UACB) 58708-110, Patos-PB, Brasil

${ }^{3}$ Professora Doutora, Disciplinas Pré clínica Multidisciplinar II, Pré clínica Multidisciplinar III e Clínica multidisciplinar I Curso de Odontologia da Universidade Federal de Campina Grande (UFCG), Unidade Acadêmica de Ciências Biológicas (UACB) 58708-110 Patos-PB, Brasil ${ }^{4}$ Professor Doutor do Curso de Engenharia de Materiais, do curso de Pós-graduação em Ciência e Engenharia de Materiais e Coordenador do Laboratório CERTBIO da Universidade Federal de Campina Grande (UFCG) 58429-900, Campina Grande - PB, Brasil

${ }^{5}$ Professor Mestre de epidemiologia das parasitoses, saúde coletiva e programas de saúde do curso de Odontologia da Universidade Federal de Campina Grande (UFCG), Unidade Acadêmica de Ciências Biológicas (UACB) 58708-110, Patos-PB, Brasil

${ }^{6}$ Acadêmica do curso de graduação em Odontologia da Universidade Federal de Campina Grande (UFCG), Unidade Acadêmica de Ciências Biológicas (UACB) 48708-110, Patos-PB, Brasi

\section{Resumo}

Introdução: A esterilização por meio da autoclave de pontas diamantadas é um procedimento seguro e essencial para garantir um resultado eficaz no procedimento odontológico. Objetivo: Avaliar a influência da esterilização de pontas diamantadas em autoclave. Material e método: A pesquisa foi desenvolvida seguindo uma sequência de pesagem em balança de alta precisão, análises em microscópio eletrônico de varredura e ciclos de esterilização. Incialmente, um grupo controle contendo cinco pontas diamantadas 1015 (Microdont) novas e não esterilizadas foram pesadas e analisadas em microscópio eletrônico de varredura. Posteriormente, as mesmas pontas diamantadas foram submetidas à cinco ciclos de esterilização em autoclave e novamente pesadas e avaliadas microscopicamente. Em seguida, as mesmas pontas foram submetidas à mais cinco ciclos de esterilização, totalizando dez ciclos, e mais uma vez pesadas e analisadas em microscópio eletrônico de varredura. As fotomicrografias foram avaliadas qualitativamente e os dados quantitativos da pesagem foram tabulados e submetidos à análise estatística. Resultados: A análise qualitativa demonstrou que as pontas diamantadas sofreram alterações estruturais, sendo àquelas submetidas a um maior número de ciclos as que apresentaram maior corrosão. De acordo com a análise estatística da pesagem, não foi observada diferença no peso das mesmas nos três momentos analisados $(\mathrm{p}=0,956)$. Conclusão: A esterilização em autoclave acarretou danos às pontas diamantadas, ocorrendo alteração estrutural do aglutinante e recobrimento dos grãos de diamantes, porém não alterou o peso das mesmas.

Descritores: Instrumentos Odontológicos; Esterilização; Microscopia Eletrônica de Varredura.

\section{Abstract}

Introduction: In order to assure a safe procedure, the sterilization of the diamond burs is essential, however, it may structurally affect the cutting instruments and generate negative consequences for the result of the dental procedure, and autoclaving has excelled as a sterilization method of these instruments. Objective: To evaluate the influence of the autoclave sterilization of diamond burs. Methodology: The research was developed following a weighing sequence in a high-precision scale, scanning electron microscope analysis and sterilization cycles. Initially, control groups containing five new and non-sterilized 1015 diamond burs (Microdont) were weighed and analyzed using scanning electron microscope. Subsequently, the same diamond burs were submitted to five autoclave sterilization cycles and then were again weighed and microscopically evaluated. Then, the same burs were submitted to five more sterilization cycles, totaling ten cycles, and once again were weighed and analyzed using scanning electron microscope. The photomicrographs were evaluated qualitatively and the data was evaluated qualitatively and the quantitative data of the weighing were tabulated and submitted to statistical analysis. Results: The qualitative analysis demonstrated that the diamond burs suffered structural alterations, and those submitted to a higher number of cycles were the ones which presented greater corrosion. According to the statistical analysis of the weighing, a difference was not observed in the weight of the burs in the three moments analyzed $(p=0,956)$. Conclusion: It is concluded that the autoclave sterilization entailed structural damage to the diamond burs, occurring corrosion of the binder and coating of the diamond grains, however did not alter their weights.

Descriptors: Dental Instruments; Sterilization; Microscopy, Electron, Scanning.

\section{Resumen}

Introducción: La esterilización por medio de la autoclave de puntas diamantadas es un procedimiento seguro y esencial para garantizar un resultado eficaz en el procedimiento odontológico. Objetivo: Evaluar la influencia de la esterilización de puntas diamantadas en autoclave. Metodología: La búsqueda ha sido desarrollada siguiendo una secuencia de pesaje en balanza de alta precisión, análisis en microscopio electrónico de exploración y ciclos de esterilización. Inicialmente, un grupo control de cinco puntas diamantadas 1015(Microdont) nuevas y no esterilizadas han sido pesadas y analizadas en microscopio electrónico de exploración. Posteriormente, las mismas puntas diamantadas han sido sometidas a cinco ciclos de esterilización en autoclave y de nuevo pesadas y evaluadas. En seguida, las mismas puntas fueran sometidas a más cinco ciclos de esterilización, totalizando diez ciclos, y más una vez pesadas y analizadas en microscopio electrónico de exploración. Las fotomicrografías han sido evaluadas cualitativamente y los dados cuantitativos del pesaje se han tabulado y sometidos a análisis estadístico. Resultados: La búsqueda cualitativa ha demostrado que las puntas diamantadas han sufrido alteraciones estructurales, siendo aquellas sometidas a más grande número de ciclos las que presentaron más grande corrosión. De acuerdo con la análisis estadístico de pesaje, no ha sido observado diferencia en el peso de las mismas en los tres momentos analizados ( $\mathrm{p}=0,956)$. Conclusión: Se concluye que la esterilización en autoclave ha ocasionados daños estructurales a las puntas diamantadas, ocurriendo corrosión del aglutinante y recubrimiento de los granos de diamantes, sin embargo no ha cambiado el peso de las mismas.

Descriptores: Instrumentos Dentales; Esterilización; Microscopía Electrónica de Rastreo.

\section{INTRODUÇÃO}

Pontas diamantadas são instrumentos rotatórios de uso rotineiro na prática odontológica, utilizadas principalmente na odontologia restauradora para confecção de preparos cavitários ${ }^{1,2}$.
A esterilização dos instrumentos rotatórios utilizados na odontologia é fundamental para evitar infecção cruzada e embora deva ser estimulado o uso desses instrumentos de forma descartável, a re-esterilização é uma prática comum 
nos consultórios odontológicos. Dentre os métodos de esterilização mais conhecidos, o uso da autoclave tem sido o que aponta melhores resultados com relação ao custobenefício, porém é necessário que seja realizada de forma adequada e a reutilização do instrumento seja limitado para garantir a qualidade final do procedimento realizado ${ }^{3}$.

Esses processos podem afetar as características morfológicas das pontas diamantadas, gerando perda de diamantes, alterando sua capacidade de corte e reduzindo a sua vida útil. A esterilização em autoclave tem sido apontada como o método que resulta em maiores alterações morfológicas nos instrumentos diamantados ${ }^{4}$. Apesar disso, devido à suas características de: eficiência, agilidade e dimensões reduzidas dos equipamentos necessários, continua a ser o método mais utilizado atualmente ${ }^{5}$.

$\mathrm{O}$ uso de pontas diamantadas que sofreram alterações morfológicas pode gerar consequências, alterando o resultado final do procedimento. Pode modificar sua eficiência de desgaste; acarretar um superaquecimento, tendo como consequência alterações pulpares ${ }^{6}$.E além disso, influenciar a qualidade final do procedimento restaurador, alterando a adesão entre dentina-resina e favorecer posteriormente a presença de infiltração ${ }^{7}$.

Pelo fato do uso da autoclave se destacar atualmente e pela necessidade da utilização de pontas diamantadas em bom estado de conservação, é preciso conhecer o efeito desse método de esterilização sobre as pontas diamantadas. A presente pesquisa tem como objetivo avaliar a influência da esterilização de pontas diamantadas em autoclave, visando identificar possíveis alterações causadas a esses instrumentos rotatórios.

\section{MATERIAL E MÉTODO}

Este estudo se trata de uma pesquisa transversal, exploratória, quali-quantitativa e experimental. Para a pesquisa utilizou-se os seguintes materiais: 5 pontas diamantadas esféricas 1015 (Microdont - São Paulo/SP, Brasil), balança de alta precisão (Adventurer/Ohaus Barueri/SP, Brasil), microscópio eletrônico de varredura (TM-1000/Hitachi - Chiyoda-ku/Toquio, Japão), autoclave (Kavo Autoclave 21L - Joinville/SC, Brasil), grau cirúrgico (Medstéril - São Paulo/SP, Brasil).

A pesquisa foi desenvolvida em uma instituição de ensino superior. As esterilizações ocorreram no centro de esterilização da clínica escola da Universidade Federal de Campina Grande (UFCG) na cidade de Patos/PB, as pesagens em balança analítica ocorreram no Laboratório Multiusuário de Pesquisas Ambientais na UFCG campus Patos/PB e as análises microscópicas ocorreram no Laboratório de Avaliação e Desenvolvimentos de Biomateriais do Nordeste da UFCG localizado na cidade de Campina Grande/PB.

A pesquisa foi executada seguindo algumas etapas. $\mathrm{Na}$ etapa inicial, o grupo controle composto por cinco pontas diamantadas novas, ou seja, sem uso e não submetidas à ciclos de esterilização foram pesadas em balança de alta precisão e em seguida analisadas no Microscópio Eletrônico de Varredura (MEV) utilizando-se os aumentos de 150x e 500x. Essas pontas diamantadas forma identificadas da seguinte forma: ponta $1(\mathrm{P} 1)$; ponta 2 $(\mathrm{P} 2)$; ponta $3(\mathrm{P} 3)$; ponta $4(\mathrm{P} 4)$ e ponta $5(\mathrm{P} 5)$ para que a comparação inicial e final ocorresse da forma correta entre as pontas estudadas. Após a realização das análises iniciais, as mesmas pontas diamantadas passaram por cinco ciclos de esterilização em autoclave a $121^{\circ} \mathrm{C}$ durante 24 minutos e na sequência foram pesadas em balança de alta precisão e analisadas no MEV.

Depois dessas etapas, as mesmas pontas diamantadas foram submetidas a mais cinco ciclos de esterilização em autoclave a $121^{\circ} \mathrm{C}$ durante 24 minutos, finalizando 10 ciclos de esterilização. Posteriormente, essas pontas diamantadas foram mais uma vez pesadas e analisadas no MEV. Após essas últimas análises, os resultados obtidos da pesagem das brocas foram tabulados e analisados e as fotomicrografias obtidas por meio do MEV nos três diferentes momentos foram analisadas e comparadas. Estas etapas da metodologia encontram-se descritas na Figura 1.

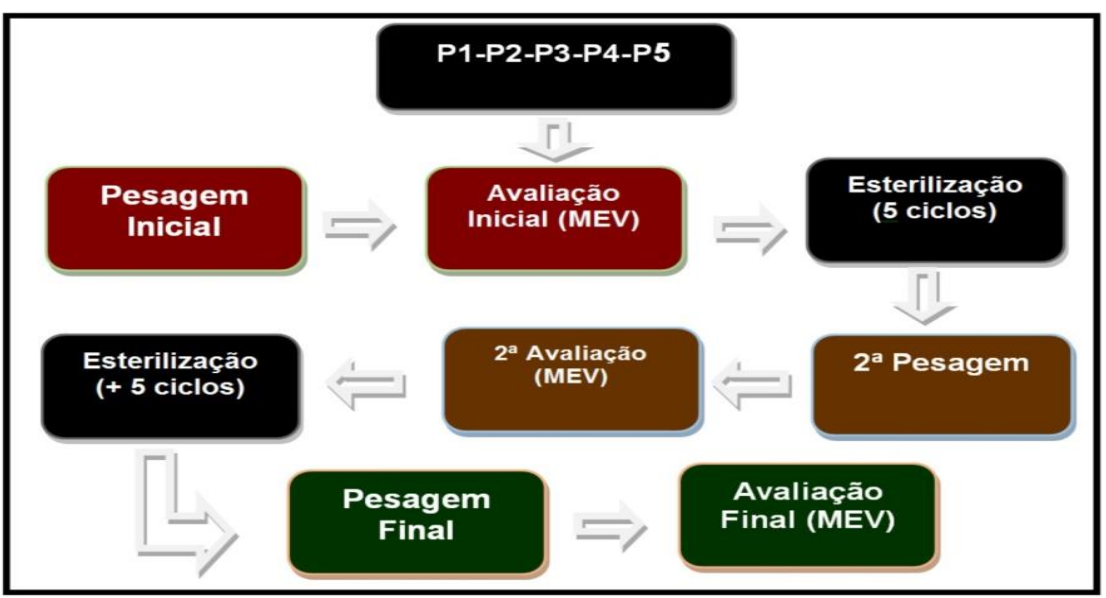

Figura 1: Fluxograma das etapas realizadas na metodologia.

*Fonte: dados da pesquisa, 2017

As fotomicrografias foram analisadas qualitativamente e comparadas e os dados quantitativos obtidos através das pesagens das pontas diamantadas foram analisados e submetidos à análise estatística, por meio do teste de Shapiro - Wilk a 5\% de significância.

\section{RESULTADOS}

Serão demonstradas as imagens representativas obtidas por meio da microscopia eletrônica de varredura, comparando as pontas diamantadas não esterilizadas (T0) àquelas submetidas à cinco (T5) e dez ciclos de esterilização (T10) nos aumentos de $150 \mathrm{x}$ e $500 \mathrm{x}$, além das pesagens das pontas também nesses três momentos (T0, T5 e T10), seguido de sua análise estatística.

Pela análise qualitativa através da comparação das fotomicrografias das pontas diamantadas observou-se que após cinco ciclos de esterilização todas as cinco brocas sofreram alteração estrutural, com cobertura parcial dos grãos de diamantes (Figura 2B e 3B), havendo uma piora dessas características após os dez ciclos de esterilização (Figura $2 \mathrm{C}$ e $3 \mathrm{C}$ ). Todas as pontas diamantadas não submetidas ao processo de esterilização em autoclave não apresentaram alterações morfológicas. (Figura 2A e 3A).


Figura 2: Fotomicrografias da ponta diamantada 4 em aumento de $150 \mathrm{x}$ 2.A. Ponta diamantada nova, sem esterilização. 2.B. Ponta diamatada após cinco ciclos de esterilização em autoclave. 2.C. Ponta diamantada após dez ciclos de esterilização.

*Fonte: dados da pesquisa, 2017 


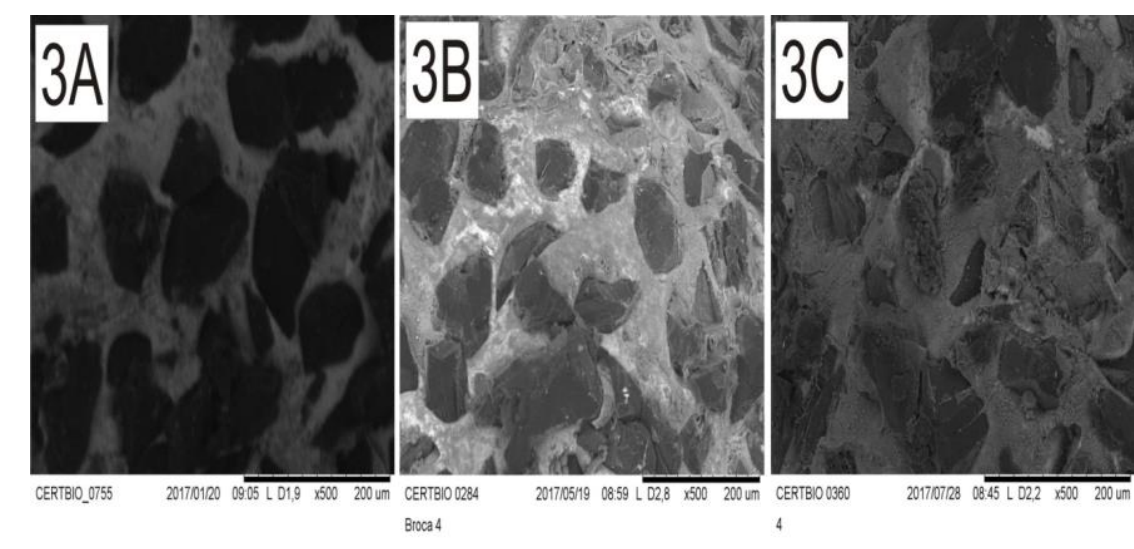

Figura 3: Fotomicrografias da ponta diamantada 4 em aumento de $500 \mathrm{x}$ 3A. Ponta diamantada nova, sem esterilização. 3B. Ponta diamatada após cinco ciclos de esterilização em autoclave. 3C. Ponta diamantada após dez ciclos de esterilização.

*Fonte: dados da pesquisa, 2017

Os dados quantitativos obtidos da pesagem das pontas diamantadas foram tabulados e analisados estatisticamente. A princípio foi realizada uma análise quantitativa exploratória a fim de verificar a normalidade das amostras pelo teste Shapiro-Wilk a 5\% de significância. Como todos os grupos apresentaram distribuição normal, foi realizada uma comparação de médias utilizando a análise de variação a $5 \%$. As análises foram feitas no programa computacional Biostat 5.0.

A Tabela 1 demonstra os valores das pesagens de P1, $\mathrm{P} 2, \mathrm{P} 3, \mathrm{P} 4$ e $\mathrm{P} 5$ antes de serem submetidas às esterilizações (T0), após cincos ciclos de esterilização (T5) e após os dez ciclos (T10). E ao final, evidencia a média dessas pesagens nos três momentos distintos e o valor final de $\mathrm{p}(\mathrm{p}=0.956)$, comprovando que o fator esterilização não interfere no peso das pontas diamantadas.

Tabela 1. Comparação dos valores da pesagem das pontas diamantadas sem esterilização, após cinco esterilizações e após dez ciclos

\begin{tabular}{c|cccc} 
& $\begin{array}{c}\text { Sem } \\
\text { esterilizaça o }\end{array}$ & $\begin{array}{c}\text { Após 5 ciclos de } \\
\text { esterilização }\end{array}$ & $\begin{array}{c}\text { Após } 10 \text { ciclos de } \\
\text { esterilização }\end{array}$ & \\
\cline { 2 - 5 } P1 & 0,2519 & 0,2521 & 0,2521 & \\
P2 & 0,2528 & 0,2528 & 0,2527 & \\
P3 & 0,2502 & 0,2503 & 0,2502 & \\
P4 & 0,2516 & 0,2514 & 0,2511 & \\
\hline P5 & 0,2525 & 0,2522 & 0,2521 & $p$ \\
Média (desvio & 0.2518 & $0.2518( \pm 0.0010)$ & $0.2516( \pm 0.0010)$ & 0,956 \\
padrão) & $( \pm 0.0010)$ & & &
\end{tabular}

*Fonte: dados da pesquisa, 2017

A Figura 4 apresenta um gráfico no qual estão demonstradas as médias dos pesos das pontas diamantadas em cada um dos três momentos (T0, T5, T10), com seus desvios-padrões para mais e para menos, comprovando mais uma vez não haver diferença entre o peso das pontas diamantadas e o fato delas não terem sido esterilizadas (T0), terem sido esterilizadas 5 vezes (T5) ou dez vezes (T10).

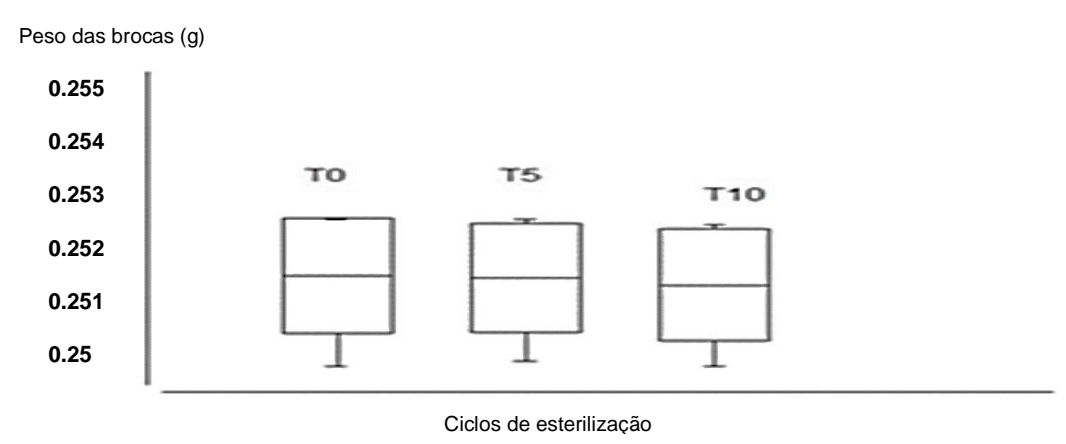

Figura 4: Comparação das médias do peso das pontas diamantadas sem esterilização (T0), após cinco esterilizações (T5) e após dez ciclos (T10). *Fonte: dados da pesquisa, 2017

\section{DISCUSSÃO}

Tanto os pacientes como os profissionais estão sujeitos a contaminação durante a realização dos procedimentos odontológicos, por este motivo a conduta dos cirurgiões dentistas deve ser sempre de forma adequada para evitar infecção cruzada nos consultórios odontológicos. A esterilização é imprescindível e deve ser realizada sempre da forma mais adequada ${ }^{8,9}$.

Sajjanshetty et al. ${ }^{3}$ avaliaram alguns métodos de descontaminação e esterilização e concluíram que nenhum método foi absolutamente eficaz na sua função, visto que mesmo a esterilização em autoclave só foi capaz de reduzir em $80 \%$ os Streptococcus mutans, $76 \%$ os Lactobacilus $76 \%$ e 74\% Candida albicans, o que pode ser explicado pelo fato de não ter sido realizada as etapas prévias à esterilização como lavagem e descontaminação das pontas diamantadas.

Porém, os métodos de esterilização podem afetar estruturalmente os materiais odontológicos, em especial as pontas diamantadas utilizadas na odontologia principalmente para preparos cavitários. É o que afirma Simamoto-Júnior et al. ${ }^{4}$, que em seu estudo avaliou a perda estrutural de pontas diamantadas pelos métodos de esterilização em autoclave, estufa e desinfecção em glutaraldeído $2 \%$, afirmando que todos esses métodos modificam estruturalmente os instrumentos de corte testados.

O presente estudo avaliou as alterações estruturais das pontas diamantadas devido a esterilização em autoclave, não comparando a outros métodos e não avaliando essa perda devido ao uso. Pela comparação das fotomicrografias obtidas, observou-se que as pontas diamantadas, após serem submetidas aos cinco e dez ciclos de esterilização em autoclave, sofrem alteração estrutural do aglutinante presente nas pontas diamantadas, fazendo com que este recobrisse os grãos de diamantes e não continuassem mais expostos. Observou-se um aumento dos danos à medida que se adicionou mais ciclos de esterilização, o que corrobora com estudos de Bianchi et al. ${ }^{10}$ e Gonçalves et al. ${ }^{11}$.

Bianchi et al. ${ }^{10}$ afirmaram que a autoclave é um método de esterilização que afeta estruturalmente as pontas diamantadas, pois estes instrumentos são constituídos por níquel que funcionam como uma ancoragem para os grãos de diamantes e essa ancoragem sofre dilatação devido ao aumento de temperatura da autoclave, o que forma um espaço entre o níquel e o grão de diamante, onde ocorre infiltração de vapor d'água, já que esse processo é um processo de esterilização úmido. Esse vapor d'água quando esfriado provoca corrosão do níquel, fazendo com que o instrumento perca então a ancoragem do diamante, reduzindo a capacidade de retenção dos grãos de diamante e consequentemente a perda destes. Devido a esse processo, a esterilização em autoclave culmina em rápida degradação das pontas diamantadas, fazendo-as perder sua capacidade de corte desde o primeiro ciclo. Mesmo a autoclave sendo o método de esterilização que mais causa alterações estruturais às pontas diamantadas, esse é um método considerado eficaz para esterilização das pontas diamantadas.

Gonçalves et al. $^{11}$ realizaram um estudo piloto utilizando três pontas diamantadas, sendo uma sem esterilização (controle), outra ponta submetida à cinco ciclos de esterilização em autoclave e por último, mais uma ponta, sendo essa submetida à dez ciclos em autoclave. Após comparação em microscopia eletrônica de varredura, observou-se que todas as pontas que passaram pela esterilização em autoclave apresentaram desgaste, sendo aquela submetida a dez ciclos, a que apresentou maior corrosão. 
Cirurgiões dentistas que responderam ao questionário de Cavalcanti et al. ${ }^{12}$ compartilham dessa mesma opinião, indicando que acreditam que o processo de esterilização acelera o envelhecimento das pontas diamantadas.

Enquanto isso, Pinelli et al. ${ }^{13}$ em sua pesquina analisaram o efeito da esterilização por estufa e autoclave em pontas diamantas, comparando-os com a ausência de esterilização, afirmando não haver diferença estatisticamente significante entre elas, mostrando que o desgaste que ocorre nas pontas diamantadas não depende do método de esterilização, sendo o tempo de uso o responsável por afetar negativamente o desempenho desses instrumentos.

Para Boldieri et al. ${ }^{14}$, os métodos de esterilização por estufa e também por autoclave não influenciam na efetividade do corte das pontas diamantadas. Nesse estudo foi avaliada a influência dos métodos de esterilização em pontas diamantadas convencionais e ultra-sônicas, tendo como resultado que as pontas convencionais apresentam mais alterações estruturais quando comparada às ultrasônicas, mas que essas alterações ocorreram apenas no grupo em que eram submetidas também ao preparo cavitário. Nos grupos em que as pontas ultra-sônicas e convencionais eram submetidos apenas aos métodos de esterilização, não houve alterações estruturais. Afirmando que a realização dos preparos cavitário é o causador da perda de diamantes.

Haddah Filho et al. ${ }^{15}$ em um estudo avaliando a capacidade de corte da lima do sistema Protaper após o uso e com esterilizações intercaladas; observou por meio da pesagem dos canais radiculares simulados que essas etapas por várias vezes provocou a perda de corte das limas, concluindo que esses instrumentos devem ser descartados, principalmente a partir do sétimo uso, devido à redução da capacidade de corte.

Porém, os resultados obtidos na presente pesquisa não condizem com os achados de Pinelli et al. ${ }^{13}$ e Boldieri et al. ${ }^{14}$, visto que se observou alterações estruturais nas pontas diamantadas submetidas à esterilização, entretanto, esta pesquisa analisou o desgaste estrutural das pontas diamantadas baseadas nos ciclos de esterilização em autoclave, sem avaliar a perda de corte da mesma. Sugere-se estudos futuros analisando também a perda de corte destas, assim como comparações com diferentes marcas e tipos de esterilização.

Há controversas a respeito da consequência da utilização das pontas diamantadas inadequadas durante os procedimentos odontológicos. Para Borges et al. ${ }^{16}$, a utilização dessas pontas com perda de diamantadas não influencia na presença de infiltrações marginais das restaurações em resina composta. O que é contrariado por Malekipour et al. ${ }^{17}$ que asseguram que o uso de pontas diamantadas desgastadas, com pouca eficiência de corte, acarreta efeitos negativos em restaurações de resina composta, pois resultam em um aumento na microinfiltração destas.

Lise et al. ${ }^{7}$ também asseguram que o uso de pontas diamantadas desgastadas para o preparo de cavidade interfere na adesão entre dentina e esmalte, podendo gerar infiltrações. Esse fato pode ser explicado devido à corrosão, desgaste e/ou perda de diamantes das pontas diamantadas.

Tendo em vista os resultados obtidos nessa pesquisa, evidencia-se a necessidade do descarte das pontas diamantadas a partir da quinta utilização, pois estas já apresentam alterações estruturais e podem afetar o resultado final do procedimento.

\section{CONCLUSÃO}

As pontas diamantadas submetidas a cinco e dez ciclos de esterilização quando analisadas em MEV e comparadas ao grupo controle mostraram ter sofrido danos estruturais, o que se mostrou mais evidente no grupo submetido a um maior número de ciclos de esterilização. Os grãos de diamantes ficaram parcialmente recobertos devido a alteração sofrida pela substância aglutinante, comprovando o prejuízo acarretado às pontas devido a esterilização em autoclave. Observou-se ainda que que não houve variação no peso das cinco pontas diamantadas antes e após os ciclos de esterilização.

\section{REFERÊNCIAS}

1. Machado AC, Tolentino AB, Spini PHR, Gonzaga RCQ, Zeola LF, Reis BR et al. Análise das dimensões padrões das macrogeometrias de pontas diamantadas de diferentes fabricantes e modelos. Rev Odontol Bras Central. 2014;23(64):47-52.

2. Soares PV, Tolentino AB, Faria VLG, Gonzaga RCQ, Spini PHR, Machado AC Influência do Modelo e Marca de Ponta Diamantada na Resistência Flexural. Rev Odontol Bras Central. 2013; 21(60):28-32.

3. Sajjanshetty S, Hugar D, Hugar S, Ranjan S, Kadani M. Decontamination methods used for dental burs - a comparative study. J Clin Diagn Res. 2014; 8(6):ZC39-41.

4. Simamoto-Júnior PC, Soares CJ, Rodrigues RB, Veríssimo C, Dutra MC, Quagliatto PS et al. Comparison of different wear burs after cavity preparation and sterilization methods. Rev Odontol Bras Central. 2012; 21(59):547-52.

5. Scariot LL, Calza JV. Condutas de Biossegurança Utilizada por Cirurgiões-Dentistas da Cidade de Marau, RS. J Oral Invest. 2015; 4(2):18-24.

6. Aranha VMS, Silva-Concílio LR, Neves ACC, Barcellos DC, Rode SM. Comparação da variação da temperatura produzida por dois tipos de instrumentos cortantes rotatórios diamantados no preparo cavitário. Braz Dent Sci. 2012; 14(3/4):38-41.

7. Lise DP, Monteiro Júnior S, Gondo R. Influence of reused dental burs on bond strength to dentin. JRD. 2014; 2(3):235-42.

8. Carmo MRC, Weckwerth PH, Chavasco JK, Franzolin SOB, Beijo LA, Cruz JRS. Risks of infection on odontological procedures. J Dent Oral Hyg. 2012; 4(4):44-50.

9. Ascari RA, Vidori J, Moretti CA, Perin EMF, Silva OM, Buss E. O processo de esterilização de materiais em serviços de saúde: uma revisão integrativa. / Braz J Surg Clin Res. 2013; 4(2):33-8.

10. Bianchi EC, Silva EJ, Cezar FAG, Aguiar PR, Bianchi ARR, Freitas CA et al. Aspectos microscópicos da influência dos processos de esterilização em pontas diamantadas. Mat Res. 2003; 6(2):203-210.

11. Gonçalves IMC, Jucá TW, Figueiredo CHMC, Penha ES, Fook MVL, Medeiros LADM. Avaliação por meio da microscopia eletrônica de varredura de alterações sofridas em pontas diamantadas-estudo piloto. Rev Uningá. 2017; 31(1):19-23.

12. Cavalcanti AN, Santos PRB, Oliveira JC, Ramos DL, Fontes CM. Conduta clínica profissional quanto ao uso e descarte de pontas diamantadas. Rev Bahiana de Odontologia. 2013; 4(1):18-26.

13. Pinelli LAP, Silva RHBT, Santana IL, Fais LMG, Guaglianoni DG. Estudo fotomicrográfico das 
características de superfície de pontas diamantadas: efeito do método de esterilização por estufa ou autoclave. Rev Odontol Univ Cid São Paulo. 2008; 20(2): 154-9.

14. Boldieri T, Bussaneli DG, Rodrigues JA, Cardoso KS, Josgrilberg EB, Cordeiro RCL. Influence of sterilization process on cutting effectiveness and durability of ultrasonic tips. Braz DenT Sci. 2015; 18 (3):10-16.

15. Haddad Filho MS, Hanbitante SM, Rosa LCL, Salum G, Almeida ETDC, Medeiros JMF. Perda de corte da lima protaper quanto à esterilização e número de uso. Rev Odontol Univ Cid São Paulo. 2011; 22(3):228-37.

16. Borges AB, Cavalcanti BN, Tavares ACS, Claro FA, Araújo MAM, Valera MC. Avaliação do desgaste de pontas diamantadas e sua influência na infiltração marginal de restaurações de resina composta. Cienc Odontol Bras. 2003; 6(1):36-43.

17. Malekipour MR, Shirani F, Tahmourespour S. The Effect of Cutting Efficacy of Diamond Burs on Microleakage of Class V Resin Composite Restorations Using Total Etch and Self Etch Adhesive Systems. J Dent. 2010; 7(4):218-25.

\section{CONFLITO DE INTERESSES}

Os autores declaram não haver conflitos de interesse.

\section{AUTOR PARA CORRESPONDÊNCIA}

Luanna Abílio Diniz Melquiades de Medeiros

luannaabiliod@gmail.com

Submetido em 18/09/2017 Aceito em 18/10/2017 